\title{
Endothelial Microparticles and Blood Coagulation Activation in Head and Neck Cancer Patients Undergoing Radiotherapy or Radiochemotherapy
}

\author{
EWA SIERKO ${ }^{1,2}$, MONIKA SOBIERSKA $^{1,3}$, EWA ZABROCKA $^{4}$, MARTA MYŚLIWIEC $^{1}$, \\ JOANNA KRUSZEWSKA ${ }^{1}$, ALINA LIPSKA ${ }^{3}$, PIOTR RADZIWON ${ }^{3}$ and MAREK Z. WOJTUKIEWICZ ${ }^{1}$ \\ ${ }^{1}$ Department of Oncology, Medical University of Bialystok, Bialystok, Poland; \\ ${ }^{2}$ Department of Radiotherapy, Comprehensive Cancer Center, Bialystok, Poland; \\ ${ }^{3}$ Regional Centre for Transfusion Medicine, Bialystok, Poland; \\ ${ }^{4}$ Department of Medicine, Stony Brook University, Stony Brook, NY, U.S.A.
}

\begin{abstract}
Background/Aim: Endothelial microparticles (EMP) are small vesicles which are released from the endothelium and contribute to blood coagulation activation in various clinical settings. The aim of this study was to examine whether EMP influence blood coagulation activation in cancer patients during radiotherapy/ radiochemotherapy $(R T / R C T)$. Materials and Methods: Sixteen head and neck cancer (HNC) patients undergoing $R T / R C T$ and 10 controls were examined. EMP and thrombin-antithrombin complex (TAT) were measured by flow cytometry and enzyme-linked immunosorbent assay (ELISA), respectively. Tissue factor-positive EMP $\left(\mathrm{TF}^{+} \mathrm{EMP}\right)$ were defined as $\mathrm{CD} 31^{+} / \mathrm{CD} 142^{+} / C D 42 b^{-}$. Results: $\mathrm{TF}^{+} \mathrm{EMP}$ were significantly elevated in $H N C$ patients before RT/RCT ( $\left.T_{0}\right)(1299 \pm 1154 / \mu l)$, one day after $R T / R C T\left(T_{1 d}\right)(1257 \pm 603 / \mu l)$ and 3 months after RT/RCT $\left(T_{3 m}\right)(1289 \pm 372 / \mu l)$ compared to controls $(688 \pm 647 / \mu l)$. $T F^{+}$EMP levels at $T_{0} / T_{1 d}$ and $T_{0}$, as well as at $T_{1 d}$ and $T_{3 m}$ were not significantly different. TAT levels at $T_{0}$ and $T_{1 d}$ did not differ significantly but at $T_{3 m}$ were significantly lower compared to $T_{0}$ and $T_{1 d} . T F^{+} E M P$ and TAT concentrations were not significantly correlated at $T_{0}(r=0.058 ; p=0.828)$, $T_{1 d}(r=0.373, p=0.154)$ and $T_{3 m}(r=-0.302, p=0.204)$. Conclusion: $T F^{+}$EMP may not contribute to hemostatic abnormalities in HNC patients.
\end{abstract}

This article is freely accessible online.

Correspondence to: Ewa Sierko, Department of Oncology, Medical University in Bialystok, Ogrodowa 12, 15-027 Bialystok, Poland. Tel: +48 856646734, Fax: +48 856646783, e-mail: ewa.sierko@iq.pl

Key Words: Endothelial microparticles, thrombin-antithrombin complex, tissue factor, head and neck cancer, coagulation activation.
Endothelial microparticles (EMP) are small vesicles released from the endothelium upon activation or apoptosis (1). Endothelium-derived microparticles represent about 10-15\% of the total microparticles (MP) in blood of healthy individuals. Their concentration ranges from 1 to $70 \times 10^{3} / \mathrm{ml}$ and their diameter varies from 0.1-2 $\mu \mathrm{m}(2,3)$. Endothelial microparticles are characterized by the expression of adhesion molecules specific for mature endothelial cells (ECs), such as CD31 (platelet-endothelial cell adhesion molecule, PECAM), CD62E (E-selectin), CD54 (intracellular adhesion molecule-1, ICAM-1) and CD62P (P-selectin). They also express CD105 (endoglin, a proliferationassociated protein), CD144 (vascular endothelial-cadherin), CD146 (S endo 1, an endothelial junctional protein), von Willebrand Factor (vWF), and phosphatidylserine (PS) (2, 3). A number of in vitro studies have demonstrated that EMP are formed after EC stimulation by inflammatory cytokines (including tumor necrosis factor - TNF- $\alpha$ ), bacterial lipopolysaccharides, reactive oxygen species, plasminogen activator inhibitor, thrombin, camptothecin and C-reactive protein (CRP) $(4,5)$. Endothelial microparticles released from activated or apoptotic EC are emerging as useful markers for detection of ECs dysfunction $(6,7)$.

The EMP level in circulating blood depends on sex (higher levels are observed in women), menstrual cycle phase and presence of some pathologic conditions (1). In vivo, EMP were found in the peripheral blood of patients with cardiovascular disorders, such as acute coronary syndrome, atherosclerosis and congestive heart failure. In addition, EMP levels were altered in diseases well known to be accompanied by ECs dysfunction, such as metabolic syndrome, obesity, diabetes mellitus, stroke, sepsis, and advanced stages of renal failure $(6,8,9)$. Moreover, higher level of EMP was reported in hematological disorders e.g. lupus anticoagulant, sickle cell disease, antiphospholipid syndrome and venous thromboembolism $(10,11)$. 
The current standard of treatment for newly diagnosed head and neck cancer (HNC) patients is surgery followed by radiotherapy (RT) or radiochemotherapy (RCT), RT alone or concomitant RCT. However, during RT not only cancer cells but also normal tissues (among them - ECs localized in the irradiated volume) are exposed to high-energy ionizing radiation and, as a result, patients experience symptoms associated with tissue damage for few weeks, months or years after RT. Early (acute) postradiation reaction (e.g. dermatitis, mucositis) is associated with inflammation and cytokinemediated responses (12). The symptoms produced by radiotherapy occur 2-3 weeks into the treatment with the greatest intensity at the end of treatment and after its completion. They usually resolve 6-8 weeks after the treatment and they disappear up to 3 months in most cases (12). Oropharyngeal and laryngeal cancer patients treated with RT were found to be at higher risk of developing venous thromboembolism (VTE) compared to their radiation-spared counterparts $(13,14)$. The prevalence of VTE among HNC patients undergoing surgery is $1.4-5.8 \%$, and can be as high as $13 \%$ when asymptomatic VTE cases are included (15). There is growing evidence that TF enriched-EMP could be a potential marker of procoagulant state in diseases associated with damage of blood vessel endothelium $(3,16,17)$. Nevertheless, there is no data concerning the RT/RCT influence on EMP formation in vivo as well as the potential impact of EMPs on blood coagulation activation in HNC patients during RT/RCT.

The aim of the study was to determine the potential EMP contribution to hemostatic abnormalities in $\mathrm{HNC}$ patients undergoing RT/RCT.

\section{Materials and Methods}

Blood samples were obtained from sixteen HNC patients diagnosed with squamous cell carcinoma in IIB-IVA clinical stages before RT (no acute radiation), one day after its completion (clinically exacerbated radiation-induced inflammation) and 3 months after the treatment (when the inflammation is resolved). Patients with conditions known or suspected to increase EMP level such as lupus anticoagulant, sickle cell disease, antiphospholipid syndrome, venous thromboembolism, metabolic syndrome, obesity, diabetes mellitus, stroke, sepsis, and advanced stages of renal failure were excluded. All patients suffered from mild hypertension. Patients were treated with RT alone, adjuvant RT or RT combined with chemotherapy (cisplatin $100 \mathrm{mg} / \mathrm{m}^{2}$ every 21 days or $40 \mathrm{mg} / \mathrm{m}^{2}$ every 1 week). Intensity modulated radiotherapy (IMRT) with high energy 6MV photons to a total dose of 60-67.5 Gy in 30 fractions (2-2.25 Gy per day) was administered. Patients' characteristics are shown in Table I. Written informed consent was obtained from the patients and the study protocol was approved by the Bioethics Committee of Medical University in Bialystok, Poland, according to the Guidelines for Good Clinical Practice (approval number - RI-002/376/2010). Control group consisted of 10 healthy individuals (7 females and 3 males).

Five $\mathrm{ml}$ of venous blood were collected in Monovette - Sarstedt tubes containing 3.8\% sodium citrate (Becton Dickinson, Franklin
Table I. Baseline characteristics of head and neck cancer patients undergoing radiotherapy/radiochemotherapy.

\begin{tabular}{lcc}
\hline Clinical factors & $\mathrm{n}=16$ & $\%$ \\
\hline Gender & & \\
Female & 5 & 31 \\
Male & 11 & 69 \\
Age (years) & & \\
31-50 & 2 & 12 \\
51-70 & 13 & 82 \\
>70 & 1 & 6 \\
Localization & & \\
Lip, tongue & 2 & 12.5 \\
Floor of oral cavity and palate & 4 & 25 \\
Hyopharynx & 3 & 19 \\
Tonsil & 2 & 12.5 \\
Indeterminate part of oral cavity & 5 & 31 \\
CS & & \\
II B & 1 & 6 \\
III & 4 & 25 \\
IV A & 11 & 69 \\
Grade & & \\
G1 & 2 & 12.5 \\
G2 & 12 & 75 \\
G3 & 2 & 12.5 \\
Treatment & & \\
RT alone & 7 & 44 \\
RCT & 9 & 56 \\
\hline
\end{tabular}

G: Grade; CS: clinical stage according to TNM classification; RT: radiotherapy; RCT: radiochemotherapy.

Lakes, NJ, USA) using 21-gauage needles (Becton Dickinson) from all patients. Two steps of centrifugation were used in order to isolate EMP from whole blood. Firstly, blood samples were centrifuged for $15 \mathrm{~min}$ at $500 \mathrm{~g}$ (removal of erythrocytes and leukocytes) to prepare platelet-rich plasma (PRP). The PRP was further centrifuged for $5 \mathrm{~min}$ at $14,000 \mathrm{~g}$ to obtain platelet-poor plasma (PPP). Both centrifugations were performed at room temperature $\left(+24^{\circ} \mathrm{C}\right)$, to avoid activation of platelets and plug generation at lower temperature. Plasma samples were not frozen because of harmful influence of low temperature on EMP vitality. The storage time of samples never exceeded $1 \mathrm{~h}$ after venipuncture. Endothelial microparticles were defined as particles bearing antigens such as CD31, CD62E, measuring below $1.5 \mu \mathrm{m}$, and were quantified with flow cytometry using two panels of monoclonal antibodies (CD31/CD62E/CD42b and CD31/CD62E/CD142). Monoclonal antibody directed to CD42b was used to exclude EMP released from platelets and megakaryocytes, whereas anti-CD142 was required for detection of tissue factor (TF)-bearing EMP.

The following monoclonal antibodies purchased from Becton Dickinson, were used in the study: CD31 stained with FITC, CD62E stained with APC, PE-Cy5-labeled anti-human CD42b, PElabeled anti-human CD142 and kappa isotype control: FITC-labeled mouse IgG1, APC-labeled mouse IgG, PE-Cy5-labeled mouse IgG1, PE-labeled mouse IgG1. Moreover, Cell WASH, fluorescenceactivated cell sorting (FACS) lysing solution, PBS wash buffer, FACS Flow Sheath Fluid, and FACS lysing solution were utilized in the study. 


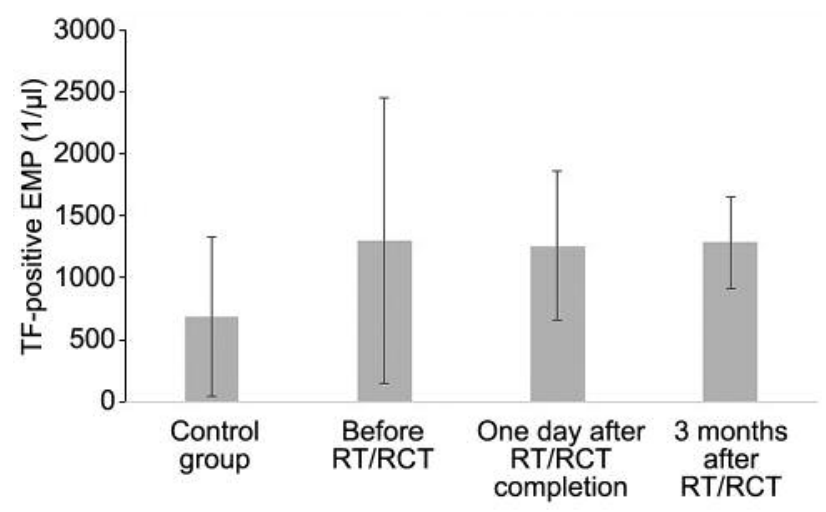

Figure 1. Levels of tissue factor (TF)-expressing endothelial microparticles (EMP) in the blood of head and neck cancer patients $(H N C)$ undergoing radiotherapy or radiochemotherapy $(R T / R C T)$ and in the control group of healthy individuals.

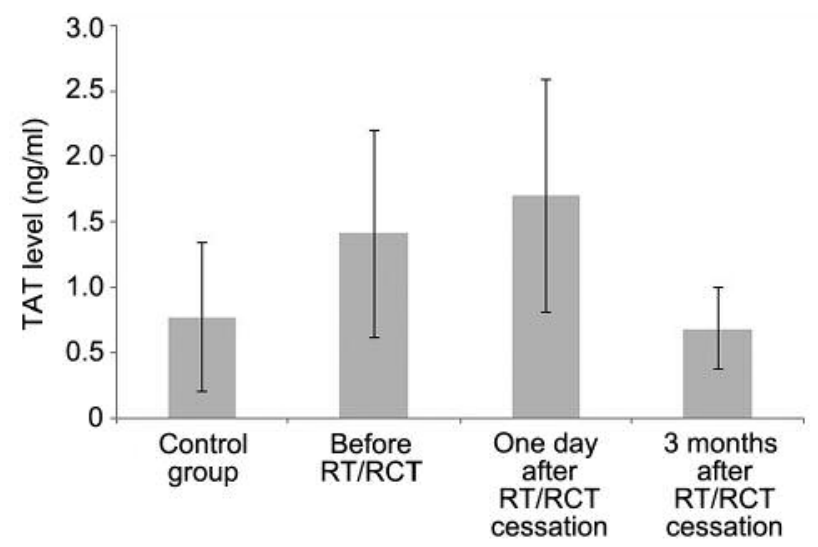

Figure 2. Levels of plasma thrombin-antithrombin complex (TAT) of head and neck cancer patients undergoing radiotherapy or radiochemotherapy $(R T / R C T)$ and in the control group of healthy individuals.

Aliquots of PPP $(30 \mu \mathrm{l})$ were incubated with $10 \mu \mathrm{l}$ of monoclonal antibodies in the dark. After $20 \mathrm{~min}$ of incubation at room temperature, the samples were gently shaken (orbital shaker, $120 \mathrm{rpm}$ ) and phosphate-buffered saline (PBS) was added. Samples of $18 \mu \mathrm{l}$ each were aspirated and counted in a $30-\mathrm{sec}$ run at medium setting. The detection of particles was triggered by a fluorescent signal greater than a predetermined value. Light scatter and fluorescence channels were set at logarithmic gain. The fluorescencepositive particles were further separated on another histogram based on size (forward light scatter). Flow cytometer counts were converted to a number of EMP per $\mu 1$. Endothelial microparticles were analyzed by flow cytometry FACS Calibur (Becton Dickinson) in Regional Centre for Transfusion Medicine in Bialystok, Poland, according to a protocol used in a previous study (18).

To assess thrombin-antithrombin (TAT) plasma complex concentration (indicating activation of blood coagulation), IMUBIND TAT (American Diagnostica, Stamford, CT, USA) was used. Five-milliliter samples of venous blood from HNC patients
Table II. Levels of tissue factor (TF)-expressing endothelial microparticles (EMP) in the blood of head and neck cancer patients (HNC) undergoing radiotherapy or radiochemotherapy (RT/RCT) and in the control group of healthy individuals.

\begin{tabular}{lcc}
\hline Time of assessment & \multicolumn{2}{c}{ EMPCD31+/CD142+/CD42b-(quantity/ul) } \\
\cline { 2 - 3 } & $\mathrm{x} \pm \mathrm{SD}$ & $\mathrm{Me}$ \\
\hline Control group & $688 \pm 647$ & 345 \\
Before RT/RCT & $1299^{*} \pm 1154$ & 1480 \\
One day after RT/RCT & $1257^{*} \pm 603$ & 1212 \\
3 months after RT/RCT & $1289^{*} \pm 372$ & 1235 \\
\hline
\end{tabular}

CD31: EMP surface antigens; CD142: tissue factor; CD42b: platelet and megakaryocyte surface antigens; $\mathrm{X}$ : mean value; SD: standard deviation; Me: median. *Statistically significant difference in comparison to control group.

undergoing RT/RCT were obtained (before treatment, one day after its completion and 3 months after the treatment) and collected in Monovette - Sarstedt tubes containing 3.8\% sodium citrate (Becton Dickinson). Blood samples were centrifuged for $15 \mathrm{~min}$ at $1500 \times g$ to receive plasma, which in the next step was frozen $\left(\leq-20^{\circ} \mathrm{C}\right)$.

Statistical analysis. Given the non-normal distribution (Shapiro-Wilk test) of the EMP concentrations, values were expressed as mean, median and standard deviation. The Wilcoxon rank sum test for dependent data was used to evaluate significance between EMP levels in different points of the study. Correlation between TF-positive EMP and TAT was measured using Spearman test. Statistical significance was defined as $p$-value $<0.05$. Analyses were performed with the statistical package STATISTICA 10 and Microsoft Excel 2010.

\section{Results}

TF-bearing EMP in HNC patients. Tissue factor positive EMP concentration was significantly higher in plasma of $\mathrm{HNC}$ patients before RT/RCT $(1299 \pm 1154 \mathrm{EMP} / \mu \mathrm{l})$, one day after RT completion $(1257 \pm 603 \mathrm{EMP} / \mu \mathrm{l})$ and 3 months after the treatment $(1289 \pm 372 \mathrm{EMP} / \mu \mathrm{l})$ compared to healthy participants $(688 \pm 647 \mathrm{EMP} / \mu \mathrm{l})(p<0.05)$ (Figure 1). Interestingly, there was no significant difference between the levels of TF-bearing EMP after RT/RCT completion and pre-treatment levels. Moreover, there was no significant difference in TF-bearing EMP levels one day after RT/RCT completion compared to 3 months after the treatment (Table II).

TAT concentration in HNC patients. Plasma TAT concentration in HNC patients was significantly higher before RT/RCT $(1.41 \pm 1.79 \mathrm{ng} / \mathrm{ml})$ in comparison to control group $(0.77 \pm 0.57$ $\mathrm{ng} / \mathrm{ml})$. There was no significant difference in TAT level before and one day after RT/RCT $(1.7 \pm 0.89 \mathrm{ng} / \mathrm{ml})$. Plasma TAT level 3 months after RT/RCT completion was significantly lower $(0.68 \pm 1.31 \mathrm{ng} / \mathrm{ml})$ in comparison to TAT concentration before treatment and one day after its completion $-p<0.05$ (Figure 2). 
A - Before RT/RCT

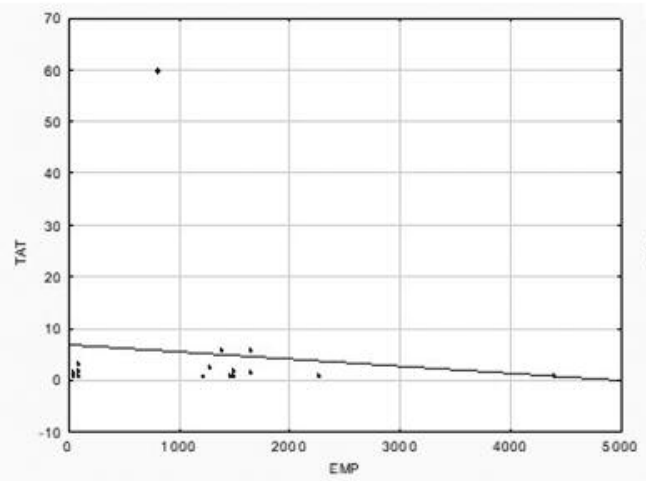

B- One day after RT/RCT

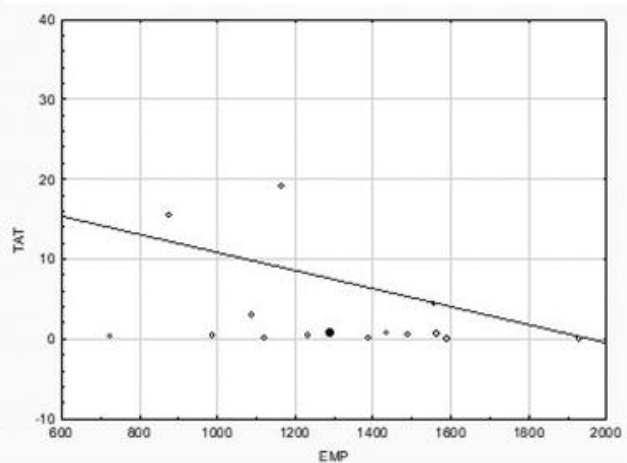

C-3 months after RT/RCT

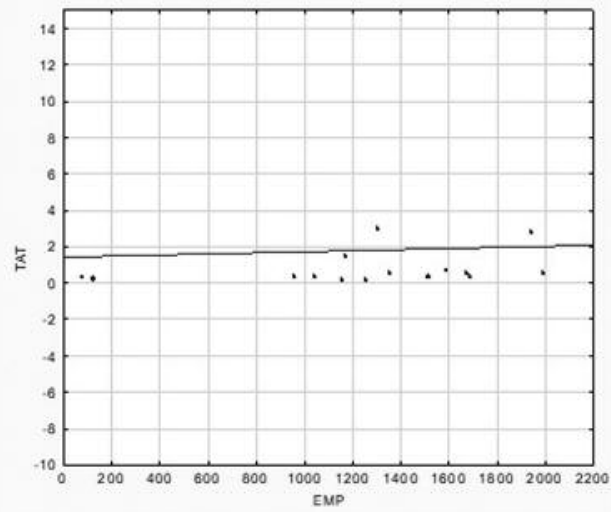

Figure 3. Correlation between tissue factor-positive endothelial microparticles (EMP) levels and concentration of thrombin-antithrombin complex (TAT) in head and neck cancer patients undergoing radiotherapy/radiochemotherapy (RT/RCT).

TF-positive EMP and TAT. There was no significant correlation between the level of TF-positive EMP (CD31+/CD142+/CD42-) and TAT concentration in the blood of HNC patients before RT/RCT ( $\mathrm{r}=0.058, p=0.828)$, one day after RT/RCT ( $\mathrm{r}=0.373, p=0.154)$ and 3 months after the treatment completion $(\mathrm{r}=-0.302, p=0.204)$ (Figure 3).

\section{Discussion}

The activation of blood coagulation in HNC patients undergoing $\mathrm{RT} / \mathrm{RCT}$ is most likely multifactorial and involves inflammation, platelet recruitment, delayed reendothelialization and overexpression of TF (19). Aside from VTE, blood coagulation can contribute to a variety of other pathologic processes, such as metastasis, tumor growth and tumor angiogenesis $(20,21)$.

Endothelium-derived microparticles are proven to play an important role in the pathogenesis of thrombotic disorders via multiple ways, e.g. TF expression (main procoagulant in cancer) $(10,22)$ and providing negatively charged phospholipid surface $(3,22)$. Phospholipids present on EMP surface facilitate binding of coagulation factors and promote the formation and activity of coagulation enzyme complexes $(3,22,23)$. Injury or stimulation of ECs contribute to TF exposure to coagulation factor VII, with subsequent TF/VIIa complex formation and initiation of coagulation cascade leading to thrombin generation and fibrin formation (10). Endothelial microparticles enriched with TF are also involved in procoagulant response $(10,24)$. Furthermore, TF-positive EMP exhibit endothelial adhesive molecules, which allows for binding to other cell types, such as monocytes and platelets, and likely enables TF transferring onto their surface $(25,26)$. In addition, EMP carry von Willebrand Factor (vWF), an adhesive protein which interacts with platelets and contributes to initiation and progression of thrombus formation (27).

It has been proven that TF-positive vesicles released from endothelium contribute to hypercoagulable state in cancer (28). Ionizing radiation also contributes to blood coagulation activation (29). A study of Szotowski and colleagues (30) showed that ionizing radiation of 5-10 Gy increased the 
release of EMP-associated TF from human umbilical vein endothelial cells (HUVEC) in vitro. However, in our study the level of TF-bearing EMP in HNC patients remained unchanged after RT/RCT. The reason for these conflicting results remains unclear, however, it is possible that higher TF-positive EMP concentrations are found mainly in irradiated tissues and not necessarily in peripheral blood. The dose of radiation can also play a role, as our patients were treated with 2-2.5 Gy per day which is 2.5-5 times less than a single radiation dose in the above-mentioned in vitro study. Unfortunately, there is no clinical data on RT influence on TF-positive EMP release, therefore our results could not be compared to other studies performed in clinical setting.

Compared to healthy individuals, the levels of TF-bearing EMP were higher in HNC patients both before and after $\mathrm{RT} / \mathrm{RCT}$, which is consistent with the findings of a study by Campello et al. (31) showing higher levels of EMP and TFpositive MP in cancer patients than in healthy controls. Although TF-bearing MP levels were reported to be higher in cancer patients with a diagnosis of VTE than without it (31), it remains unclear whether elevated TF-positive MP levels are a cause or a consequence of VTE. Given that thrombin inhibitors were found to prevent the increases in circulating tumor-induced TF-positive MPs (32), thrombin generated during VTE event may be one of the factors contributing to TF-positive MPs release.

Interestingly, the present study revealed no correlation between TF-positive EMP and TAT levels before and after $\mathrm{RT} / \mathrm{RCT}$ suggesting that EMP may not contribute to blood coagulation activation. Of note, EMP also express anticoagulant factors such as thrombomodulin and protein $\mathrm{C}$ receptors (33), therefore, the overall coagulable properties of EMP in cancer patients may depend on the balance between pro- and anticoagulation factors found within EMP (34).

Importantly, EMP subpopulations expressing various antigens require distinct methods of their isolation from the whole blood (centrifugation protocol) which makes it difficult to compare the results of various studies. Namely, EMP formed as a result of ECs activation via TNF- $\alpha$ stimulation are characterized by expression of inducible endothelial markers, such as CD62E, whereas EMP released during apoptosis are more likely to constitutively express endothelial cell markers - CD31 $(2,5)$.

In conclusion, our study does not provide evidence for $\mathrm{RT} / \mathrm{RCT}$ influence on rapid procoagulant EMP release or EMP contribution to the activation of blood coagulation in HNC patients undergoing RT/RCT. Further research and study group enlargement is needed to define whether concentrations of EMP in HNC patients are sufficient to initiate or augment blood coagulation.

\section{Conflicts of Interest}

The Authors declare no conflicts of interest regarding this study.

\section{Authors' Contributions}

Ewa Sierko - Concept of the study, supervision, interpreting results, writing the manuscript, approval of the text of the manuscript. Monika Sobierska - collecting material, performing study, interpreting the results, writing the manuscript, performing experiments, approval of the manuscript. Ewa Zabrocka - interpreting the results, writing the manuscript, performing experiments, approval of the manuscript. Marta Myśliwiec - performing experiments, literature searching, approval of the manuscript. Joanna Kruszewska - performing experiments, literature searching, approval of the manuscript. Alina Lipska - performing experiments, approval of the manuscript. Piotr Radziwon - supervision on the experiments, approval of the manuscript. Marek Z. Wojtukiewicz - concept of the study, supervision, approval of text of manuscript.

\section{References}

1 Sierko E, Sokół M and Wojtukiewicz MZ: Endothelial microparticles (EMP) in physiology and pathology. Postepy Hig Med Dosw (Online) 69: 925-932, 2015. PMID: 26400879, DOI: $10.5604 / 17322693.1165195$

2 Dignat-George F and Boulanger CM: The many faces of endothelial microparticles. Arterioscler Thromb Vasc Biol 31: 27-33, 2011. PMID: 21160065, DOI: 10.1161/ATVBAHA. 110.218123

3 Hussein MNA, Böing AN, Biró E, Hoek FJ, Vogel GMT, Meuleman DG, Sturk A and Nieuwland R: Phospholipid composition of in vitro endothelial microparticles and their in vivo thrombogenic properties. Thromb Res 121: 865-871, 2008. PMID: 17889282, DOI: 10.1016/j.thromres.2007.08.005

4 Hussein MN, Meesters EW, Osmanovic N, Romijn M, Nieuwland $\mathrm{R}$ and Sturk A: Antigenic characterization of endothelial cell-derived microparticles and their detection ex vivo. J Thromb Haemost 11: 2434-2443, 2003. PMID: 14629480, DOI: 10.1046/j.1538-7836.2003.00455.x

5 Jimenez JJ, Jy W, Mauro LM, Soderland C, Horstman LL and Ahn YS: Endothelial cells release phenotypically and quantitatively distinct microparticles in activation and apoptosis. Thromb Res 109: 175-180, 2003. PMID: 12757771, DOI: 10.1016/S0049-3848(03)00064-1

6 Amabile N, Guerin AP, Leroyer A, Mallat Z, Nguyen C, Boddaert J, London GM, Tedgui A and Boulanger CM: Circulating endothelial microparticles are associated with vascular dysfunction in patients with end-stage renal failure. $\mathrm{J}$ Am Soc Nephrol 16: 3381-3388, 2005. PMID: 16192427, DOI: 10.1681/ASN.2005050535

7 Morel O, Toti F, Morel N and Freyssinet JM: Microparticles in endothelial cell and vascular homeostasis: are they really noxious? Haematologica 94: 313-317, 2008. PMID: 19252173, DOI: 10.3324/haematol.2009.003657

8 Boulanger N, Amabile AP, Guérin B, Pannier AS, Leroyer Z, Mallat CN, Tedgui A and London GM: In vivo shear stress determines circulating levels of endothelial microparticles in endstage renal disease. Hypertension 49: 902-908, 2007. PMID: 17309952, DOI: 10.1161/01.HYP.0000259667.22309.df

9 Gonzalez-Quintero VH, Smarkusky LP, Jimenez JJ, Mauro LM, Jy W, Hortsman LL, O’Sullivan MJ and Ahn YS: Elevated plasma endothelial microparticles: preeclampsia versus gestational hypertension. Am J Obstet Gynecol 191: 1418-1424, 2004. PMID: 15507976, DOI: 10.1016/j.ajog.2004.06.044 
10 Combes V, Simon AC, Grau GE, Arnoux D, Camoin L, Sabatier F, Mutin M, Sanmarco M, Sampol J and Dignat-George F: In vitro generation of endothelial microparticles and possible prothrombotic activity in patients with lupus anticoagulant. J Clin Invest 104: 93 102, 1999. PMID: 10393703, DOI: 10.1172/JCI4985

11 Liu ML and Williams KJ: Microvesicles: potential markers and mediators of endothelial dysfunction. Curr Opin Endocrinol Diabetes Obes 19: 121-127, 2012. PMID: 22248645, DOI: 10.1097/MED.0b013e32835057e9

12 Stone H, Coleman NC, Anscher MS and McBride WH: Effects of radiation on normal tissue: consequences and mechanisms. Lancet Oncol 9: 529-536, 2003. PMID: 12965273, DOI: 10.1016/S14702045(03)01191-4

13 Guy JB, Bertoletti L, Magné N, Rancoule C, Mahé I, Font C, Sanz O, Martín-Antorán JM, Pace F, Vela JR and Monreal M: Venous thromboembolism in radiation therapy cancer patients: Findings from the RIETE registry. Crit Rev Oncol Hematol 113: 83-89, 2017. PMID: 28427527, DOI: 10.1016/j.critrevonc.2017.03.006

14 Nobis A, Sierko E, Kasprowicz B, Jelski S, Wojtukiewicz MZ and Sierko E: Massive saddle pulmonary embolism during radiochemotherapy of head and neck cancer. Pol Arch Intern Med 127: 561-563, 2017. PMID: 28817547, DOI: 10.20452/pamw.4080

15 Tham T, Rahman L, Persaud C, Olson C and Costantino P: Venous thromboembolism risk in head and neck cancer: significance of the preoperative platelet-to-lymphocyte ratio. Otolaryngol Head Neck Surg 159: 85-91, 2018. PMID: 29406795, DOI: 10.1177/01945998 18756851

16 Hron G, Kollars M, Weber H, Sagaster V, Quehenberger P, Eichinger S, Kyrle PA and Weltermann A: Tissue factor-positive microparticles: cellular origin and association with coagulation activation in patients with colorectal cancer. Thromb Haemost 97: 119-123, 2007. PMID: 17200778

17 Piccin A: Endothelial microparticles and endothelial damage: 'The Tip and the iceberg'. Acta Haematol 132: 199-204, 2014. PMID: 24662890, DOI: 10.1159/000360600

18 Amabile N, Heiss C, Real WM, Minasi P, McGlothlin D, Rame EJ, Grossman W, De Marco T and Yeghiazarians Y: Circulating endothelial microparticle levels predict hemodynamic severity of pulmonary hypertension. Am J Respir Crit Care Med 177: 1268-1275, 2008. PMID: 18310479, DOI: $10.1164 / \mathrm{rccm}$ 200710-1458OC

19 Goldin-Lang P, Niebergall F, Antoniak S, Szotowski B, Rosenthal P, Pels K, Schultheiss HP and Rauch U: Ionizing radiation induces upregulation of cellular procoagulability and tissue factor expression in human peripheral blood mononuclear cells. Thromb Res 120: 857-864, 2007. PMID: 17337288, DOI: 10.1016/j.thromres.2007.01.008

20 Lima LG and Monteiro RQ: Activation of blood coagulation in cancer: implications for tumour progression. Biosci Rep 33: e00064, 2013. PMID: 23889169, DOI: 10.1042/BSR20130057

21 Wojtukiewicz MZ, Hempel D, Sierko E, Tucker SC and Honn $\mathrm{KV}$ : Protease-activated receptors (PARs)--biology and role in cancer invasion and metastasis. Cancer Metastasis Rev 34: 775796, 2015. PMID: 26573921, DOI: 10.1007/s10555-015-9599-4

22 Leroyer AS, Anfosso F, Lacroix R, Sabatier F, Simoncini S, Njock SM, Jourde N, Brunet P, Camoin-Jau L, Sampol J and DignatGeorge F: Endothelial-derived microparticles. Biological conveyors at the cross road of inflammation, thrombosis and angiogenesis. Thromb Haemost 104: 456-463, 2010. PMID: 20664896, DOI: 10.1160/TH10-02-0111
23 Lovren F and Verma S: Evolving role of microparticles in the pathophysiology of endothelial dysfunction. Clin Chem 59: 11661174, 2013. PMID: 23529703, DOI: 10.1373/clinchem.2012. 199711

24 Lacroix R and Dignat-George F: Microparticles as a circulating source of procoagulant and fibrinolytic activities in the circulation. Thromb Res 129: S27-29, 2012. PMID: 22424856, DOI: 10.1016/j.thromres.2012.02.025

25 Nomura S, Ozaki Y and Ikeda Y: Function and role of microparticles in various clinical settings. Thromb Res 123: 8-23, 2008. PMID: 18667228, DOI: 10.1016/j.thromres.2008.06.006

26 Lynch SF and Ludlam CA: Plasma microparticles and vascular disorders. Br J Haematol 137: 36-48, 2007. PMID: 17359370, DOI: $10.1111 / \mathrm{j} .1365-2141.2007 .06514 . \mathrm{x}$

27 Jimenez JJ, Jy W, Mauro LM, Horstman LL, Soderland C and Ahn YS: Endothelial microparticles released in thrombotic thrombocytopenic purpura express von Willebrand factor and markers of endothelial activation. Brit J Haematol 123: 896-902, 2003. PMID: 14632781, DOI 10.1046/j.1365-2141.2003.04716.x

28 Falanga A, Schieppati F and Russo D: Cancer tissue procoagulant mechanisms and the hypercoagulable state of patients with cancer. Semin Thromb Hemost 41: 756-764, 2015. PMID: 26408922, DOI: 10.1055/s-0035-1564040

29 Verheij M, Dewit LG and van Mourik JA: The effect of ionizing radiation on endothelial tissue factor activity and its cellular localization. Thromb Haemost 73: 894-895, 1995. PMID: 7482424

30 Szotowski B, Antoniak S, Goldin-Lang P, Tran QV, Pels K, Rosenthal P, Bogdanov VY, Borchert HH, Schultheiss HP and Rauch U: Antioxidative treatment inhibits the release of thrombogenic tissue factor from irradiation- and cytokineinduced endothelial cells. Cardiovasc Res 73: 806-812, 2007. PMID: 17266944, DOI: 10.1016/j.cardiores.2006.12.018

31 Campello E, Spiezia L, Radu CM, Bulato C, Castelli M, Gavasso S and Simioni P: Endothelial, platelets, and tissue factor-bearing microparticles in cancer patients with and without venous thromboembolism. Thromb Res 127: 473-477, 2011. PMID: 21256535, DOI: 10.1016/j.thromres.2011.01.002

32 Wojtukiewicz MZ, Hempel D, Sierko E, Tucker SC and Honn KV: Thrombin-unique coagulation system protein with multifaceted impacts on cancer and metastasis. Cancer Metastasis Rev 35: 213-233, 2016. PMID: 27189210 DOI: 10.1007/s 10555-016-9626-0

33 Flamant $\mathrm{S}$ and Tamarat R: Extracellular vesicles and vascular injury: new insights for radiation exposure. Radiat Res 186: 203218, 2016. PMID: 27459703, DOI: 10.1667/RR14482.1

34 Aharon A and Brenner B: Microparticles, thrombosis and cancer. Best Pract \& Res Clin Hematol 22: 61-69, 2009. PMID: 19285273, DOI: 10.1016/j.beha.2008.11.002 